\title{
Bloqueo neurolítico del tercer ganglio simpático dorsal en pacientes con síndrome de Raynaud. Una serie de casos
}

\author{
J. A. Flores Cantisani ${ }^{1}$, M. G. Hinojosa Reyes ${ }^{1}$, G. C. Palacios Saucedo², M. L. Prieto Duarte ${ }^{3}$ \\ y O. Becerra Alcántara ${ }^{4}$
}

${ }^{1}$ Servicio de Medicina de Dolor y Cuidados Paliativos. Unidad Médica de Alta Especialidad. Monterrey N.L. México. ${ }^{2}$ Departamento de Investigación. Unidad Médica de Alta Especialidad; Monterrey N.L. México. ${ }^{3}$ Médico Externo de la Especialidad en Medicina del Dolor y Cuidados Paliativos. ${ }^{4}$ Médico Externo de Anestesiología

Flores Cantisani JA, Hinojosa Reyes MG, Palacios Saucedo GC, Prieto Duarte ML, Becerra Alcántara O. Bloqueo neurolítico del tercer ganglio simpático dorsal en pacientes con síndrome de Raynaud. Una serie de casos. Rev Soc Esp Dolor 2013; 20(2): 50-54.

\begin{abstract}
Background: Raynaud's phenomenon, a vascular disorder that is common in the general population, consists of paroxysmal onset distal vasospasm in small cutaneous arteries and arterioles of extremities, usually with little systemic impact. There are anatomical and physiological bases to produce a selective sympathetic block ad using neurolytic agents in the thoracic ganglia T2 and T3 considered synaptic stations that are distributed in the upper limb.

Objective: The objective of this work was to determine the efficacy of neurolytic block of the third dorsal ganglion due to Raynaud's syndrome in a series of cases.

Material and methods: A review of records of eight cases with Raynaud's phenomenon that presented multifactorial origin were conducted on Clinic of the Pain located in the Unidad Médica de Alta Especialidad, Monterrey Nuevo León, México.

Cases: Eight patients from 41 to 72 years old were medically treated for Raynaud's phenomenon using sympathicolysis dorsal third node.

Conclusions: Raynaud's phenomenon with a prevalence ranging from 1 to $25 \%$ has an increased sympathetic activity. The ischemic pain that is determined by total or partial failure in the oxygen transport to tissues was reduced in these patients by performing a selective sympathicolysis in the thoracic ganglia T2 and T3 considered synaptic stations that are distributed
\end{abstract}

Recibido: 01-10-11.

Aceptado: 01-03-12. in the upper limb. It was demonstrated in this study that this treatment is selective with less adverse effects to the patients.

Key words: Neurolytic block. Third dorsal ganglion. Raynaud phenomenon.

\section{RESUMEN}

Introducción: El fenómeno de Raynaud es un trastorno vascular, frecuente en la población general, que consiste en la aparición paroxística de espasmo vascular distal, en pequeñas arterias y arteriolas cutáneas de las extremidades, con escasa repercusión sistémica. Existen bases anatómicas y fisiológicas para producir un bloqueo simpático selectivo utilizando agentes neurolíticos en los ganglios torácicos T2 y T3, considerados estaciones sinápticas que se distribuyen en el miembro superior.

Objetivo: Determinar la eficacia del bloqueo neurolítico del tercer ganglio dorsal en el síndrome de Raynaud de una serie de casos.

Material y métodos: Se realizó la revisión de expedientes de una serie de ocho casos con fenómeno de Raynaud de causa multifactorial, en la Clínica del Dolor de la Unidad Médica de Alta Especialidad Monterrey; Nuevo León, México.

Casos: Ocho casos de 41 a 72 años de edad con fenómeno de Raynaud, tratados médicamente, a los cuales se les realizaron simpaticolisis del tercer ganglio dorsal.

Conclusiones: El fenómeno de Raynaud tiene una prevalencia que va del 1 al 25\%, en este hay una actividad simpática aumentada por lo que al realizar una simpaticolisis selectiva se logra mejorar el dolor isquémico, el cual está determinado por la insuficiencia total o parcial en el aporte de oxígeno a los tejidos, además de que tenemos bases anatómicas y fisiológicas para limitar el bloqueo a los ganglios T2 y T3, considerados como las estaciones sinápticas que interrumpen los impulsos que se distribuyen en el miembro superior, siendo por tanto más selectivos y con menores efectos adversos para el paciente.

Palabras clave: Bloqueo neurolítico. Tercer ganglio dorsal. Fenómeno de Raynaud. 


\section{INTRODUCCIÓN}

El fenómeno de Raynaud es un trastorno vascular frecuente que consiste en la aparición paroxística de espasmo vascular distal, que afecta a pequeñas arterias y arteriolas cutáneas de las extremidades, fue descrito en 1862, tiene poca repercusión sistémica. Y su prevalencia es de 2 al $25 \%$ en hombres y 1,8 al 30\% en mujeres (1-4).

Se desconoce la causa de la fisiopatología primaria de este fenómeno; pero probablemente está relacionada con una respuesta anormal del sistema nervioso simpático, fue descrito por primera vez por Galeno en el siglo XIX y posteriormente por Claude Bernard, y finalmente en 1925 Stetlow describe el uso de alcohol en la cadena simpática cervical inferior y del primer ganglio torácico en pacientes con angina de pecho, en este mismo tiempo Leriche estudia las indicaciones del bloqueo del ganglio estrellado en pacientes con distrofia refleja postraumática con excelentes resultados. Las complicaciones que se presentaron al bloquear el primer ganglio de la cadena torácica son neumotórax, parálisis del nervio laríngeo recurrente, disfagia y síndrome de Claude Bernard Horner. Existen bases anatómicas y fisiológicas para limitar el bloqueo con sustancias neurolíticas a los ganglios T2 y T3, en dicho punto ascienden y establecen sinapsis con las fibras posganglionares, que se distribuyen en el miembro superior, evitando de esta manera el bloqueo de la cadena simpática cervical y por lo tanto complicaciones como el síndrome de Horner (5-8).

Son doce ganglios torácicos; el primer torácico se une habitualmente al último cervical formando lo que conocemos como ganglio estrellado, tienen una disposición segmentaria y son pequeños, cada ganglio se une a su respectivo nervio espinal por medio de los ramos comunicantes blancos y por los ramos comunicantes grises (9). Las fibras simpáticas entre T1 y T6, provocan la liberación de acetilcolina desde las neuronas posganglionares que estimula las glándulas sudoríparas exocrinas, por lo que la simpatectomía dorsal ha sido utilizada con éxito en el tratamiento de hiperhidrosis palmar, el síndrome de Horner se puede presentar en un $1 \%$ de los pacientes, considerablemente menor en frecuencia que si se realizara una simpaticolisis en el ganglio estrellado (10-14).

\section{MATERIAL Y MÉTODOS}

Se hizo una revisión de una serie de 8 expedientes de pacientes con síndrome de Raynaud a los cuales se les realizó un bloqueo neurolítico del tercer ganglio dorsal, como parte del tratamiento del dolor todos firmaron un consentimiento informado donde se les explicó en qué consistía el procedimiento, así como los riesgos y las complicaciones del mismo.

\section{DESCRIPCIÓN DE LA TÉCNICA DEL BLOQUEO}

Todos los procedimientos fueron bajo control fluoroscópico arco en $\mathrm{C}$, en mesa translúcida y en sala de radiología. Se colocó al paciente en posición de Rossiere modificada, con rectificación de columna dorsal alineándola con la columna cervical, se monitorizaron a todos de forma no invasiva (PANI, FC, $\mathrm{SPO}_{2}, \mathrm{FR}$ ), se canalizó un acceso venoso periférico 20 , se inició una precarga de solución cristaloide, a todos los pacientes se les colocó puntas nasales con $\mathrm{O}_{2}$ al $40 \%$, con flujo de $2 \mathrm{l} / \mathrm{min}$. Previo a la aplicación del bloqueo se les dio sedación a los pacientes con midazolam, fentanilo y propofol a dosis convencionales y adaptadas al paciente.

Para localizar el sitio de la punción se palpa entre el músculo esternocleidomastoideo y la tráquea, buscando la presencia de pulso carotídeo entre los dedos índice y medio de la mano. Encontraremos la apófisis transversa cervical más prominente, C6 (tubérculo de Chaussignac), situada a la altura del cartílago cricoides, este tubérculo está en la cara medial del ECM y en la mayoría de los pacientes aproximadamente a $1,5 \mathrm{~cm}$ de la línea media del cuello. Rechazaremos la arteria carótida y la vena yugular, así como la tráquea. De esta manera, lateralizando los vasos, dejaremos entre nuestros dedos el tubérculo de C6. Previo habón cutáneo con anestésico local (lidocaína al 2\%), se realiza presión con ambos dedos y con una aguja del calibre 22, de bisel corto y 17" de longitud, recorreremos una mínima distancia al atravesar la piel dirigiéndonos a la punta del tubérculo de C6. Después de contactar con él (sin penetrar en el periostio), aspiraremos repetidamente. Se realiza una inclinación de $35^{\circ}$ con movimientos firmes pero suaves, se desliza la aguja por el borde anterior de apófisis transversas unión con cuerpo vertebral y detrás de pleura parietal llegando al $3 .^{\text {er }}$ ganglio torácico. Se administraron $60 \mathrm{mg}$ de lidocaína al 2\% y luego con 1 a $2 \mathrm{ml}$ de medio de contraste de material no iónico de yodo se visualizó el ganglio, posteriormente se aplican $750 \mathrm{mg}$ de fenol al $15 \%(5 \mathrm{ml})$, se limpia la aguja antes de extraer con $1 \mathrm{ml}$ de lidocaína al $2 \%$ se hace presión, aproximadamente 2 minutos, posterior al retiro de la aguja para evitar hematomas. Todos los pacientes fueron internados a cargo del Servicio de Medicina del Dolor y fueron valorados al mes, tres, seis meses y un año posteriores al procedimiento.

\section{RESULTADOS}

Se revisaron los expedientes clínicos de pacientes sometidos a bloqueo neurolítico del tercer ganglio dorsal, los cuales están registrados en la estadística diaria de pacientes sometidos a procedimientos intervencionistas del departamento de Medicina del Dolor y Cuidados Paliativos de la Unidad Médica de Alta Especialidad de Monterrey (Nuevo León). 


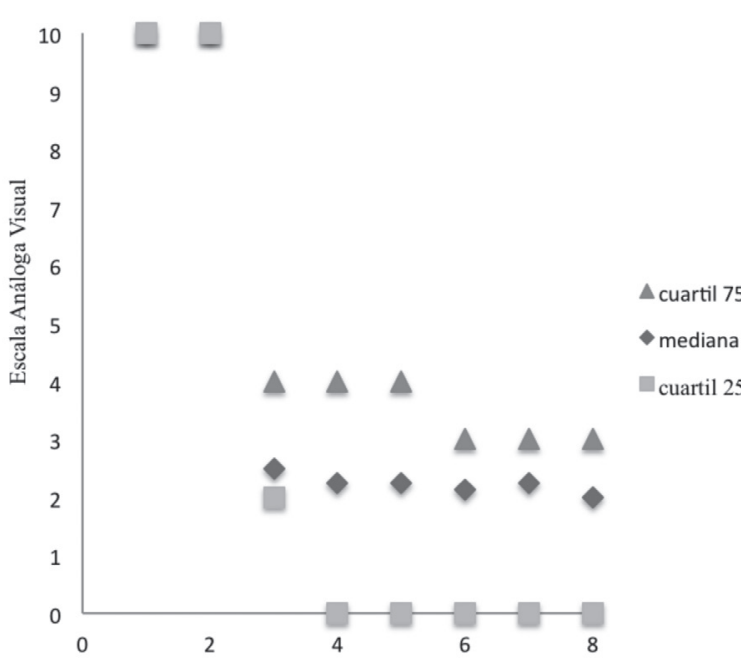

Fig. 1. Grado de dolor evaluado con la escala análoga visual (EVA) antes y después del bloqueo neurolítico en pacientes con síndrome de Raynaud. 0-2 previo al bloqueo, en el servicio de reumatología y al ingreso en medicina del dolor. 3 inmediatamente posterior.

Los 8 pacientes presentaron un dolor importante al momento de ser valorados por nuestro servicio según la escala análoga visual (EVA), que valora del 0 al 10 el grado de dolor, todos presentaron un EVA inicial de 10. El servicio de Reumatología ya los estaba tratando con pentoxifilina y antiinflamatorios no esteroideos, de inicio, en nuestro servicio, todos fueron manejados a base de opioides, se les solicitó exámenes de laboratorio y gabinete para valorar la posibilidad de un procedimiento invasivo en ellos, se les explicó el mismo y previo consentimiento informado se les realizó el procedimiento descrito previamente.

En la fig. 1 se observa el grado del dolor del paciente según la escala análoga visual (EVA), de inicio en todos los pacientes fue de 10 e inmediatamente posterior al bloqueo tuvo una mediana de 2,5, con un rango de 0 a 4 , manteniéndose prácticamente igual durante el año posterior al procedimiento.

En este grupo de ocho pacientes se observó que, además de una disminución considerable del dolor, se limitó la enfermedad, presentando una capiloroscopia normal, la cual fue valorada periódicamente por el médico reumatólogo además no hubo progresión cutánea.

\section{DISCUSIÓN}

El síndrome de Raynaud es frecuente en esclerodermia, su fisiopatología sigue sin comprenderse, lo que sí sabemos es que se encuentran implicadas anomalías estructurales

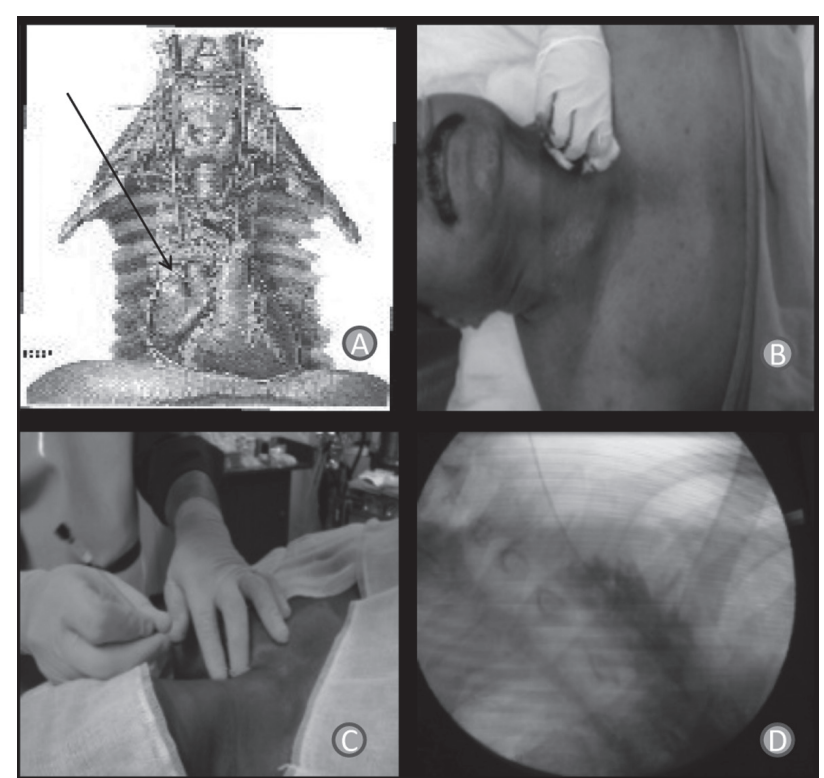

Fig. 2. Descripción anatómica y de la técnica del bloqueo neurolítico del tercer ganglio dorsal. A. Localización anatómica del tercer ganglio dorsal. B. Posición Rossiere modificada y asepsia del sitio de punción. C. Localización del sitio de punción. D. Control fluoroscópico y con medio de contraste del tercer ganglio dorsal.

en la pared vascular, incluido endotelio y músculo liso, mecanismos de control neuronal del tono vascular y mediadores circulantes. Se cree que la interrelación de todos estos factores origina apoptosis de células endoteliales y la activación de moléculas de adhesión, que producen un engrosamiento de la íntima hasta la oclusión vascular, aunando al desequilibrio entre vasoconstricción y vasodilatación, mediada por la activación de receptores de bajo umbral de activación ante la exposición a bajas temperaturas. Se generan además moléculas proinflamatorias y procoagulantes en el endotelio, así como una disminución en la producción de sustancias vasodilatadoras, como el óxido nítrico, también hay alteraciones en la fibrinolisis y agregación plaquetaria, disminución de la deformabilidad de los glóbulos rojos, aumento de la viscosidad de la sangre. Dentro de los factores de riesgos tenemos que hay relación entre el hábito tabáquico y la intensidad de la isquemia digital en pacientes con Raynaud y esclerodermia, también están implicados factores hormonales que explicarían una clara predominancia de mujeres afectadas y factores genéticos $(14,15)$.

En general, la terapia vasodilatadora es más efectiva en el fenómeno de Raynaud primario que en el secundario, quizás por un mayor daño estructural en estos últimos casos. El uso de antagonistas de los canales del calcio es el tratamiento farmacológico de primera línea, limitando la entrada de calcio dentro de las células de la musculatura lisa de las arteriolas. Las dihidropiridinas son las moléculas de referencia para el tratamiento, y entre ellas el nifedipino es la más estudiada y empleada, no solo por 
TABLA I. CARACTERÍSTICAS CLÍNICAS Y EPIDEMIOLÓGICAS DE UNA SERIE DE OCHO PACIENTES CON DOLOR QUE PRESENTAN SÍNDROME DE RAYNAUD Y FUERON SOMETIDOS A BLOQUEO NEUROLÍTICO

\begin{tabular}{lllllll}
\hline Caso & Sexo & Edad (años) & Diagnóstico & Tabaquismo & $\begin{array}{l}\text { Antecedentes } \\
\text { Patológicos }\end{array}$ & $\begin{array}{l}\text { Tiempo de } \\
\text { evolución en años } \\
\text { de enfermedad }\end{array}$ \\
\hline 1 & Femenino & 72 & Esclerodermia & Positivo & Ninguno & 11 \\
2 & Femenino & 41 & Esclerodermia & Negativo & Ninguno & 15 \\
3 & Femenino & 47 & Esclerodermia & Negativo & Ninguno & 5 \\
4 & Masculino & 52 & Esclerodermia & Positivo & Hipertensión & 2 \\
& & & arterial & \\
5 & Femenino & 57 & Esclerodermia & Positivo & Hipotiroidismo & 10 \\
6 & Femenino & 51 & Lupus & Positivo & Diabetes mellitus & 7 \\
& & & eritematoso & & & \\
7 & & sistémico & & Hipotiroidismo & 10 \\
8 & Femenino & 51 & Esclerodermia & Negativo & Ninguno & 14 \\
\hline
\end{tabular}

sus efectos vasodilatadores, sino también por su inhibición de la actividad plaquetaria. En la actualidad se prefiere el uso de preparados de liberación prolongada por una mejor tolerancia. Otras dihidropiridinas (nicardipino, amlodipino, felodipino, isradipino) y el diltiazem también se usan, disminuyendo el número e intensidad de los ataques. Su uso se limita por los efectos secundarios, como el descenso de la presión arterial, cefalea o edemas de extremidades inferiores. El uso de verapamilo no suele ser eficaz en el tratamiento del fenómeno de Raynaud. Los parches de liberación sostenida de nitratos son otra posibilidad para un alivio sintomático el fenómeno de Raynaud, pero la elevada incidencia de cefalea limita su utilización. Una alternativa es el uso de nitratos en gel de aplicación tópica.

Los inhibidores de la enzima conversora de la angiotensina presentan resultados contradictorios en el tratamiento del fenómeno de Raynaud, aunque se utilizan en pacientes con esclerodermia o hipertensión pulmonar. El losartán, antagonista del receptor tipo II de la angiotensina, sí parece ofrecer un buen resultado en el control del fenómeno de Raynaud, similar al de las dihidropiridinas Las prostaciclinas y la prostaglandina E1 son medicamentos de uso hospitalario para el fenómeno de Raynaud grave, pero tienen una escasa vida media. El iloprost, un análogo de la prostaglandina, tiene una respuesta clínica duradera a partir de su administración intravenosa durante 5 días consecutivos. No hay datos de eficacia si la administración es oral. El bosentán es un antagonista de los receptores de la endotelina, que ha demostrado su eficacia en la prevención de las úlceras cutáneas en los dedos, al tiempo que disminuye el número y frecuencia de las crisis. Los inhibidores de la fosfodiesterasa, como el sildenafilo o el vardenafilo, consiguen un efecto vasodilatador generalizado, que también actúa de forma eficaz en los dedos en el fenómeno de Raynaud, incluso mejorando las úlceras digitales en pacientes resistentes a otros tratamientos. La simpatectomía quirúrgica es considerada como última etapa en el tratamiento de este fenómeno y solo en casos graves, sin embargo con esta técnica guiada por fluoroscopia y en manos de expertos es un procedimiento adecuado en esta serie de casos, con éxito en 8 casos a largo plazo, sin complicaciones graves en este grupo de pacientes $(15,16)$.

Hay estudios basados en evidencia que han demostrado que varios fármacos como la prazosina y el ciclofenil; son eficaces en el tratamiento a corto plazo con respecto a la severidad y a la progresión a úlceras digitales, teniendo en cuenta que el comportamiento de dicho fenómeno es variable entre los pacientes y la mayoría de estudios fue hecho en muestras pequeñas y el seguimiento de pacientes solo fue a corto plazo, en la mayoría solo a seis meses (17-20).

Con el antecedente de que este tipo de pacientes presentan una actividad simpática aumentada, tenemos que si realizamos una simpaticolisis mejoraremos el dolor isquémico, determinado por la insuficiencia total o parcial en el aporte de oxígeno a los tejidos. Por un lado, la acción analgésica directa por el efecto inhibidor de las neuronas del asta dorsal de la médula espinal nos explica el doble efecto en el control del dolor de estos pacientes, además de esto se limitaran la progresión de la enfermedad $(13,21)$.

Nuestro propósito de presentar estos casos es abrir nuevas posibilidades de tratamiento en pacientes que presenten vasculitis periférica y dolor originado por el sistema nervioso autónomo, con la posibilidad de éxito casi inmediato en el control del dolor, así como una funcionalidad 
de las extremidades, asimismo la posibilidad de mejorar su pronóstico en forma significativa. Tal vez la explicación de porqué en algunas pacientes el efecto de la simpaticolisis es variable en el tiempo se deba al grado de hiperreactividad del mismo, ya que el tiempo de evolución de la enfermedad tampoco nos explicaría esto, debido a que tuvimos pacientes con 18 y 14 años de evolución a los que con un bloqueo único se autolimitaron las lesiones y se disminuyó considerablemente el dolor. Considerando todo lo anterior la simpaticolisis del tercer ganglio dorsal debería considerarse una alternativa del tratamiento de este fenómeno, segura y con menos efectos adversos que el uso de fármacos, con la posibilidad de autolimitar las lesiones y limitando la progresión de este padecimiento (1-3).

\section{AGRADECIMIENTOS}

Al personal del Departamento de Medicina del Dolor y Cuidados Paliativos de la Unidad Médica de Alta Especialidad. Monterrey-Nuevo León. México.

A la Dra. Laura Hinojosa Reyes por su apoyo en el presente trabajo.

\section{CORRESPONDENCIA:}

Martha Guadalupe Hinojosa Reyes

e-mail: marthahinojosareyes@hotmail.com

\section{BIBLIOGRAFÍA}

1. Fishman SM. En: Bonica JJ (editor). Manejo del dolor. 3er edición. 2009. p. 498-500.

2. Block JA, Sequeira W. Fenómeno de Raynaud, Lancet 2001,357:2042-8.

3. Wigley FM. Raynaud's Phenomenon. N Engl J Med 2002; 347:1001-8

4. Trafford JC, Lafferty K, Potter CE, et al. An epidemiological survey of Raynaud's phenomenon. Eur J Vasc Surg 1988; $2: 167-70$
5. Freire E, Camba MA. Técnicas e indicaciones del bloqueo del ganglio estrellado en el tratamiento del dolor. Rev Soc Esp Dolor 2002;9:328-37.

6. Terán JC, Green C, Miranda J, et al. Fenómeno de Raynaud con lesiones isquémicas digitales. tratamiento con bloqueo de ganglio estrellado, presentación de un caso. Revista El Dolor 2008:5050-2.

7. Cheshire WP, Freeman R. Disorders of sweating. Semin Neurol. 2003;23:399-406.

8. Adar R, Kurchin A, Zweig A, et al. Palmar hyperhidrosis and its surgical treatment: A report of 100 cases. Ann Surg.1977; 186:34-41

9. Drake RL, Vogl W, Mitchell AWM. Anatomía para estudiantes. Elservier España. 2000.

10. Landry GJ, Edwards JM, Porter JM. Current management of Raynaud's syndrome. Adv Surg 1996;30:333-47.

11. Miller LM, Morgan RF. Vasospastic disorders: etiology, recognition, and treatment. Hand Clin 1993; 9:171-87.

12. Seibold JR, Allegar NE. The treatment of Raynaud's phenomenon. Clin Dermatol 1994;12:317-21.

13. Biebl MO, Fraedrich G, Larcher P, et al. Amelioration of specific phobias after thoracic sympathetic block in patients with localized hyperhidrosis. Ann Thorac Surg. 2007;83:1578-9.

14. Callejas MA, Grimalt R, Cladellas E. Hyperhidrosis Update. Actas Dermosifiliogr. 2010;101(2):110-8.

15. Ceru S, Pancera S, Sansone S, et al. Effects of five days versus one day infusion of iloprost on the peripheral microcirculation in patients with systemic sclerosis. Clin Exp Rheumatol. 1997; 15:381-5.

16. Selenko-Gebauer N, Duschek G, Minimair G, et al. Successful treatment of patients with severe secondary Raynaud's phenomenon with the endothelin receptor antagonist bosentan. Rheumatology (Oxford). 2006 Oct;45 Suppl 3:iii45-8.

17. Pope J. Iloprost and cisaprost for Raynaud's phenomenon in progressive systemic sclerprogressive systemic sclerosis (Review). Cochrane. 2009.

18. Pope J. Ketanserin for Raynaud's phenomenon in progressive systemic sclerprogressive systemic sclerosis (Review). Cochrane. 2009

19. Pope J. Prazozin for Raynaud's phenomenon in progressive systemic sclerprogressive systemic sclerosis (Review). Cochrane. 2009

20. Tingey P Cyclofenil for Raynaud's phenomenon in progressive systemic sclera progressive systemic sclerosis (Review). Cochrane. 2009

21. Glaser DA, Hebert AA, Pariser DM, et al. Primary focal hyperhidrosis: scope of the problem. Cutis. 2007;79(Suppl 5):5-17. 